\title{
Incompressible paired Hall state, stripe order and the composite fermion liquid phase in half-filled Landau levels
}

\author{
E. H. Rezayi ${ }^{a}$ and F. D. M. Haldane ${ }^{b}$ \\ ${ }^{a}$ Department of Physics, California State University, Los Angeles, California 90032 \\ ${ }^{b}$ Department of Physics, Princeton University, Princeton, New Jersey 08544
}

(June 1999; revised March 24, 2000)

\begin{abstract}
We consider the lowest two Landau levels at half filling. In the higher Landau level $(\nu=5 / 2)$, we find a first-order phase transition separating a compressible striped phase from a paired quantum Hall state, which is identified as the Moore-Read state. The critical point is very near the Coulomb potential and the transition can be driven by increasing the width of the electron layer. We find a much weaker transition (either second-order or a crossover) from pairing to the composite fermion Fermi liquid behavior. A very similar picture is obtained for the lowest Landau level but the transition point is not near the Coulomb potential.
\end{abstract}

73.20.Dx, 73.40.Kp, 73.50.Jt

A two-dimensional electron gas in an intense perpendicular magnetic field displays a host of collective ground states. The underlying reason is the formation of twodimensional Landau levels in which the kinetic energy is completely quenched. In the macroscopically-degenerate Hilbert space of a given Landau level, only the Coulomb potential remains, making the system strongly interacting. The fractional quantum Hall effect [1], at rational fillings of the Landau levels, is one instance of such a ground state (GS). Other examples occur at half integral fillings of Landau levels. In the lowest Landau level, $\rho_{x x}$ shows a shallow minimum and no plateau [2] in $\rho_{x y}$. This behavior has been associated with a compressible Fermiliquid-like state [3] of composite fermions [4] (CF). In sharp contrast, a plateau in $\rho_{x y}$ and activated $\rho_{x x}$ has been observed at filling factor $\nu=5 / 2$ [5], indicative of an incompressible quantum Hall state. Above the second Landau level, for $\nu=9 / 2,11 / 2,13 / 2$, the transport is highly anisotropic [6 8], suggesting the GS is a compressible charge density wave (CDW) stripe state [9 11].

Some years ago we proposed [12] a spin-singlet wavefunction $\Psi_{\mathrm{HR}}$ for the $5 / 2$ effect based on the idea of electron pairing [13]. Moore and Read [14] (MR), building on the analogy of this state to Bardeen-Cooper-Schrieffer pairing of CF's, proposed a similar spin-polarized pairing wavefunction $\Psi_{\mathrm{MR}}$ :

$$
\begin{aligned}
& \Psi_{\mathrm{HR}}\left(\left\{z_{i}, \alpha_{i}, \beta_{i}\right\}\right)=\operatorname{Pf}_{i, j}\left[\frac{\alpha_{i} \beta_{j}-\beta_{i} \alpha_{j}}{\left(z_{i}-z_{j}\right)^{2}}\right] \Psi_{L}^{(\nu=1 / 2)}, \\
& \Psi_{\mathrm{MR}}\left(\left\{z_{i}, \alpha_{i}, \beta_{i}\right\}\right)=\underset{i, j}{\operatorname{Pf}}\left[\frac{\alpha_{i} \alpha_{j}}{z_{i}-z_{j}}\right] \Psi_{L}^{(\nu=1 / 2)},
\end{aligned}
$$

where $\alpha$ and $\beta$ are spinor coordinates for up and down spins, $\operatorname{Pf}[A]$ is the Pfaffian of an antisymmetric matrix $A$ [15], and $\Psi_{L}^{(\nu=1 / 2)}$ is the Laughlin state (for bosons).

Subsequently, Greiter, Wen and Wilczek (GWW) 16] suggested that the MR state may be a possible candidate for the $5 / 2$ effect. Recent numerical calculations by Morf [17] show the polarized state to have a lower energy than spin-singlet states even without Zeeman energy. Yet, these studies have not established what the true nature of the $5 / 2$ state is. In this paper we present evidence which suggests that the $\nu=5 / 2$ effect indeed derives from a paired state which is closely related to the MR polarized state or, more precisely, to the state obtained by particle-hole $(\mathrm{PH})$ symmetrization of the MR state. We also show why the transport may not be quantized 18 and may become anisotropic upon tilting the field, as observed [19.20]. We find a first-order phase transition from a striped phase to a strongly-paired state, after which the system evolves into a Fermi-liquid-like state, either by a continuous crossover to a weakly-paired state, or a second-order transition to a gapless state (our calculations cannot distinguish these possibilities).

Our conclusions are based on numerical studies for up to 16 electrons in two geometries: sphere and torus. The torus is particularly convenient for investigating the nature of the ground state at $\nu=1 / 2$. All three states of interest - composite fermion Fermi surface, pairing and CDW - are realized at flux $N_{\phi}=2 N$ (in units of flux quanta). This avoids a problem on the sphere, where, for a given $N$, different $\nu=1 / 2$ states occur at slightly different $N_{\Phi}$. We only consider states within a given Landau level and discard the kinetic energy. The Hamiltonian is:

$$
H=\sum_{m=0}^{\infty} V_{m} \frac{2}{N_{\Phi}} \sum_{\mathbf{q}} e^{-\frac{1}{2} q^{2}} L_{m}\left(q^{2}\right) \sum_{i<j} e^{i \mathbf{q} \cdot\left(\mathbf{R}_{i}-\mathbf{R}_{j}\right)},
$$

where $\mathbf{R}_{i}$ is the guiding center [21] coordinate of the $i^{\prime}$ th electron, $L_{m}(x)$ 's are the Laguerre polynomials, and $V_{m}$ is the energy of a pair of electrons in a state of relative angular momentum $m$. These are the pseudo-potential parameters 21,22]. The magnetic length is set to 1 . Unless otherwise specified the data presented here is for ten fully-polarized electrons in a hexagonal unit cell.

The Fermi-liquid state is well-described by a Fermi sea of composite fermions [23,24], which on the torus is [24]:

$$
\left.\left|\Psi_{C F}\left(\left\{\mathbf{k}_{\mathbf{i}}\right\}\right\rangle=\operatorname{det}_{i, j}\left[\exp \left(i \mathbf{k}_{i} \cdot \mathbf{R}_{j}\right)\right]\right| \Psi_{L}^{(\nu=1 / 2)}\right\rangle,
$$


where the $\left\{\mathbf{k}_{i}\right\}$ are distinct (and belong to the usual set of wavevectors allowed by the PBC's) and are clustered together to form a filled "Fermi sea" centered on $\mathbf{k}_{\mathrm{av}}=$ $\sum \mathbf{k}_{i} / N$. The total momentum quantum number $\mathbf{K}$ 25] is determined by the value of $\mathbf{k}_{\mathrm{av}}$ relative to the set of allowed $\mathbf{k}$ 's (the CF state is essentially left invariant by a uniform "boost" $\left\{\mathbf{k}_{i}\right\} \rightarrow\left\{\mathbf{k}_{i}+\mathbf{k}\right\}$ ), and takes one of $N^{2}$ distinct values $[25]$. There are four distinct values of $\mathbf{k}_{\mathrm{av}}$ which are invariant under $180^{\circ}$-rotation: $\mathbf{k}_{\mathrm{av}}=0$, and $\mathbf{k}_{\mathrm{av}}$ halfway between allowed $\mathbf{k}$-vectors (three distinct values which correspond to the three distinct values of $\mathbf{K}$ for the MR state on the torus).

The $\nu=1 / 2$ spin-polarized electron eigenstates of (3) have particle-hole $(\mathrm{PH})$ symmetry [26]; the CF state is almost (99.935\%) PH-symmetric and also has a large projection $(99.25 \%)$ on the exactly PH-symmetric GS of the Coulomb potential in the lowest Landau level.

The periodic MR states [16] can be obtained as the zero-energy ground states of a 3-body short range potential [16], the corrected form of which is

$$
H_{3}=-\sum_{i<j<k} \mathcal{S}_{i, j, k}\left\{\nabla_{i}^{4} \nabla_{j}^{2}\right\} \delta^{2}\left(\mathbf{r}_{i}-\mathbf{r}_{j}\right) \delta^{2}\left(\mathbf{r}_{j}-\mathbf{r}_{k}\right),
$$

where $\mathcal{S}_{i, j, k}$ is a symmetrizer. Note that (in contrast to (3)) $H_{3}$ has no PH symmetry and the MR state does not possess definite parity under $\mathrm{PH}$ transformations.

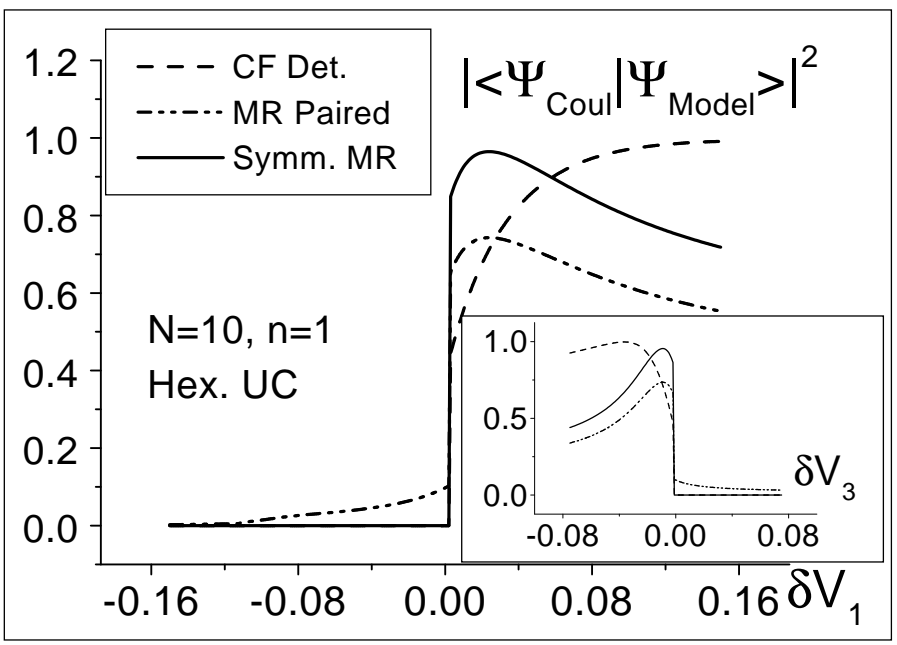

FIG. 1. The projection of the exact GS of the Coulomb interaction in the $n=1$ Landau level, plus an extra short-range pseudopotential $\delta V_{1}$ ( $\delta V_{3}$ in the inset), on the CF, MR, and PH-symmetrized MR model states. The GS PH parity changes at a level crossing near $\delta V=0$.

The nature of the ground state of (3) depends on the relative strengths of the pseudo-potentials, in particular $V_{1}$ and $V_{3}$ (even- $m$ pseudo-potentials do not affect polarized states). Figure 1 and Fig. A (below) show the projection of the $\mathrm{CF}$ and $\mathrm{MR}$ states on the exact GS in two different $\mathrm{PBC}$ geometries, as $V_{1}$ and $V_{3}$ are varied relative to their Coulomb values in the first excited Landau level $(n=1)$. Varying $V_{3}$ alone (the inset of Fig. 1) or varying both $V_{1}$ and $V_{3}$, yields similar results, though $\delta V_{1}$ has an opposite effect to $\delta V_{3}$. A study using spherical geometry [17] also identifies the phase at large $\delta V_{1}$ with the CF liquid.

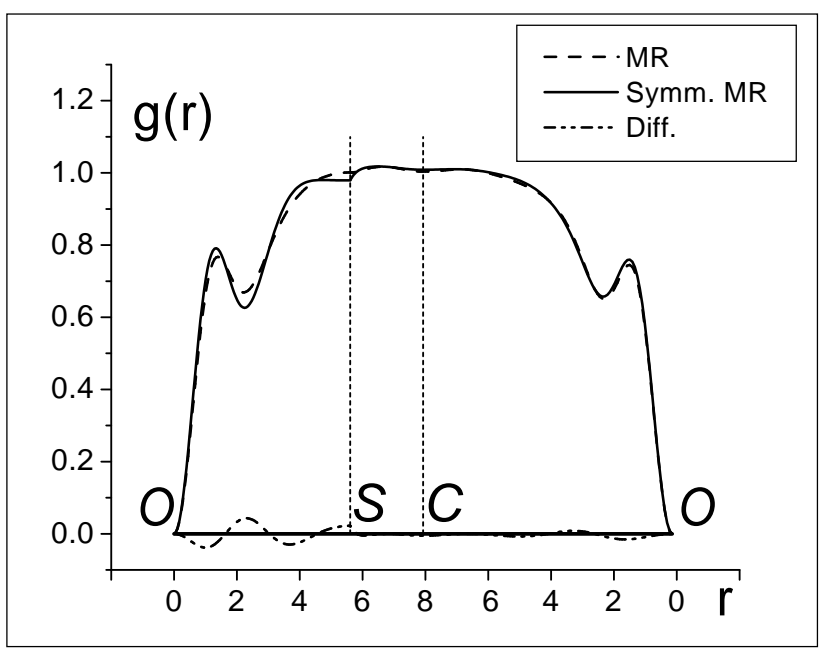

FIG. 2. The real-space pair-correlation function for the MR state and its PH-symmetrized counterpart, evaluated in the second ( $n=1)$ Landau level); their difference is also shown. $g(\mathbf{r})$ in a square unit cell is shown along a path from the origin $O$ to the midpoint of a side $S$, to a corner $C$, and back to $O$.

A first-order phase transition from a compressible state to an incompressible paired state is clearly seen. The transition is very close to the Coulomb value $\left(\delta V_{1}=\right.$ $\left.\delta V_{3}=0\right)$. We obtain similar results in the lowest Landau level, except that the transition point occurs at $\delta V_{1}=-0.092, \quad \delta V_{3}=0$ and at $\delta V_{1}=0, \quad \delta V_{3}=0.048$. Details of these studies will be given elsewhere. For both Landau levels, we only observe the strongly paired state in a narrow window. The projection of the MR state on the exact ground state does not exceed $73 \%$ in this region. However, if the MR state is first PH-symmetrized, this projection becomes $97 \%$. The two-particle correlation function $g(\mathbf{r})$ of the states before and after symmetrization is shown in Fig. 2. The paired character of the MR state is essentially unaltered (Fig. 2 shows that each electron has one particularly close partner); the near isotropy of $g(\mathbf{r})$ is characteristic of the incompressible states, and should improve with increasing system size.

An interesting feature in Fig. 1 is the absence of any obvious sharp transition from the paired state to the compressible Fermi-liquid-like $\mathrm{CF}$ state as $V_{1}$ is increased further. This is also seen in the excitation spectrum. Figure 3 shows the low-lying excitation spectrum as a function of $V_{1}$. Again, there is only one first-order level crossing transition (shown by up-arrows). The levels that cross have the same translational and $180^{\circ}$-rotation symmetry 
but belong to opposite parities under $\mathrm{PH}$ transformation. The MR state has a finite overlap with the exact GS on both sides of the transition as it has components with both $\mathrm{PH}$ symmetries. As $\delta V_{1}$ increases further, the excitation spectrum gradually evolves from having a clear gap to the compressible CF Fermi-liquidlike spectrum [23,24. The crossover is approximately at the point where the spectrum begins to change at the level crossings of the excited states (down-arrows). Similar crossover behavior is also seen on the sphere, and in those geometries on the torus where the most-compact Fermi sea has $180^{\circ}$-rotation symmetry, so the CF state has the same $\mathbf{K}$ as the MR state.

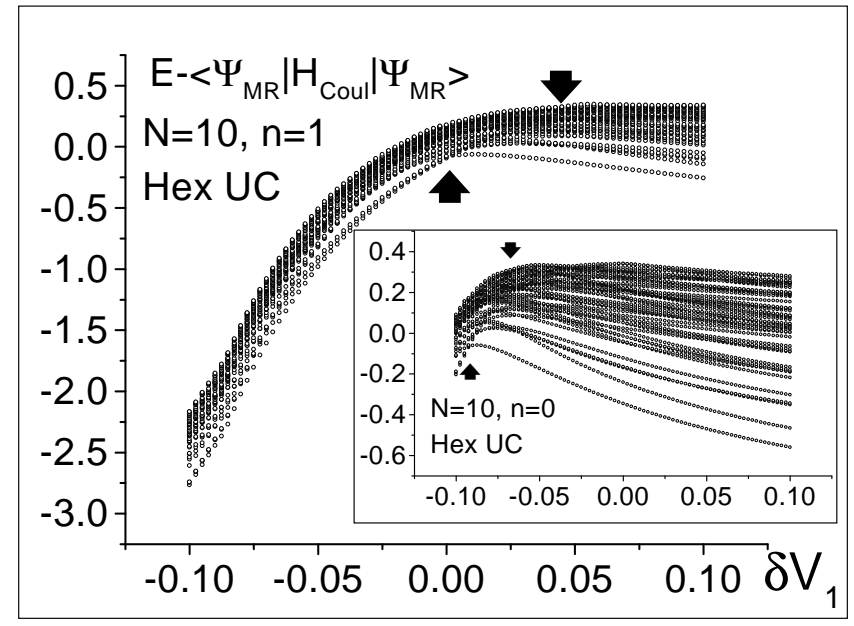

FIG. 3. The low-lying spectrum (relative to the variational energy of the MR state)plotted vs. $\delta V_{1}$ for the $n=1$; the inset shows this for the $n=0 \mathrm{LL}$. The Coulomb point is $\delta V_{1}=0$. The energies are scaled by the bandwidth of the two-particle system. The region between the arrows is the strong pairing regime.

The hallmark of compressible CF states is the sensitivity of the GS $\mathbf{K}$ to the PBC geometry. For example, the Fermi surface for 10 electrons with the square PBC does not have $180^{\circ}$-rotation symmetry and has a $\mathbf{K}$ different from the MR state. A sharp transition is seen in this case (Fig. 1). As Figs. 1 and 1 clearly demonstrate, the evolution to the CF state is strongly dependent on geometry while the transition to the striped phase is not. We believe this rules out a first-order transition to the $\mathrm{CF}$ liquid state. The picture most consistent with our studies is that, after the first-order transition to the paired state, the system may always be paired, and smoothly crosses over from a strong to a weak pairing regime as the interaction is varied. In the weak pairing regime, such a system would exhibit CF Fermi-liquid behavior at energy scales and temperatures above the gap and paired quantum Hall behavior below the gap; finite-size effects in our calculations will mask a very small gap. If true, this would eliminate the infra-red divergences of [3].
In agreement with this, we find substantial pairing character in the lowest Landau level for the Coulomb potential in both spherical and toroidal geometries. For example, on the sphere (with flux $N_{\phi}=2 N-3$ ) we found that the projections of the MR state on the exact ground state of the Coulomb potential increases with system size $(43 \%, 52 \%, 56 \%$ at $N=12,14,16)$, even though the relevant $L=0$ Hilbert space grows twenty-fold. This would be consistent with weakly-bound pairs that are larger than the linear system size at small $N$; however, because we cannot study larger $N$, we are unable to conclusively exclude the possibility of a second-order (or even a weakly first-order) phase transition to a gapless CF state.

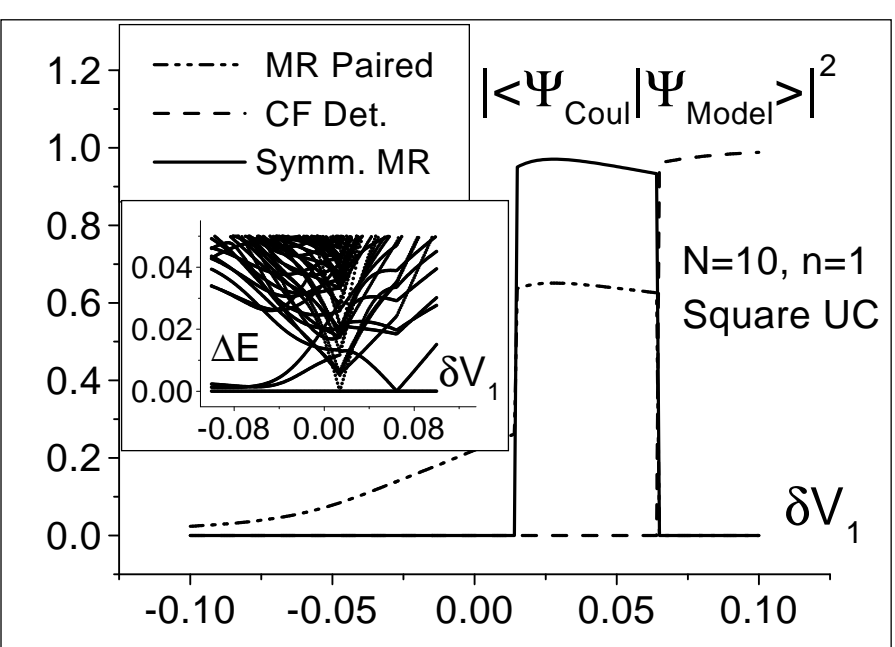

FIG. 4. Same as Fig. 1 but for a square unit cell. The inset shows the excitation spectrum (the GS energy is subtracted) as a function of $\delta V_{1}$. The transition points are marked by the collapse of the gap. In the striped phase (left portion) one recovers the typical degeneracies seen in the $n=2,3 \mathrm{LL}$ 's.

We next turn to the compressible state seen to the left of the transition in Figs. 1, 3 and 1 . To show its character more clearly, we reduce $V_{1}$ by 0.05 (about $10 \%$ of its Coulomb value). Here, as in the Fermi-liquid state, the GS $\mathbf{K}$-vector changes with size and geometry indicating the state is compressible. We now consider 12 electrons in a rectangular unit cell and tune the aspect ratio to 0.5 . We find two strong peaks in the static guiding center structure function $S_{0}(\mathbf{q})$ and in the charge susceptibility $\chi(\mathbf{q})$ with ordering wavevector $\mathbf{q}^{*}=(1.1,0)$ which constitute the signature of the CDW stripe ordering 111. This system forms three stripes and the weight of the single Slater determinant state with the occupation pattern 000011110000111100001111 is about $58 \%$. Edge fluctuations of stripes seem to be stronger here than in the higher Landau levels; $V_{1}$ has to be somewhat reduced below the transition for the characteristic degeneracies of the broken symmetry phase to be well developed (inset of Fig. (1). 
We believe that the proximity of the critical point to the Coulomb potential is the principal reason for the disappearance of the paired Hall state upon tilting the field [27]. One effect of the tilted field is to compress the 2D layer 16,28]. Indeed, we have found that varying the layer width drives this transition (as suggested by GWW [16]) in most of the pbc geometries that we have studied. The critical width varied from 0.23 to 2.4 in these systems. Fig. 5 shows the overlap (squared) as a function of the layer width in the $n=1$ Landau level. We have used the Fang-Howard model for layer profile (with $w=2 b)$ 21,29. In the lowest Landau level, the GS of the Coulomb potential is well in the CF regime. The projection on the MR state increases from $54 \%$ for a thin layer to $64 \%$ for very wide layers ( $83 \%$ on the PH-symmetrized MR state). For both Landau levels, increasing the layer width increases the pairing correlations, as seen also in Monte-Carlo calculations [30].

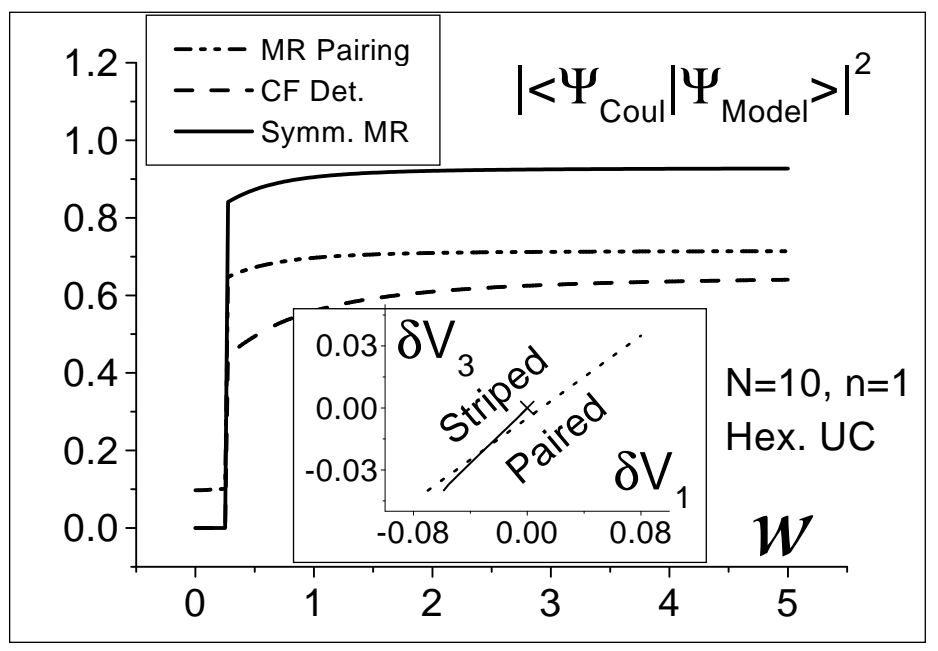

FIG. 5. The overlap squared of the two model states as the layer width $w$ is varied in the $n=1$ Landau level. The inset shows the boundary between striped and paired phases and how layer thickness changes $\delta V_{1}$ and $\delta V_{3}$ as $w$ is varied from 0 (at the cross) to 1 . The system crosses the phase boundary at $w=0.3$ along the solid line.

We acknowledge useful discussions with R. Morf, J. Eisenstein, and especially N. Read. This work was supported by NSF DMR-9420560 (E.H.R.) and DMR9809483 (F.D.M.H.). We thank ITP-UCSB for their hospitality during the "Disorder and Interactions in Quantum Hall and Mesoscopic Systems" workshop supported by NSF-PHY94-07194.

Note added-A realistic potential taking into account finite layer width, screening by filled Landau levels and tilted field effects, including mixing of subband levels, (modeled for Eisenstein's experimental samples and supplied to us by Girvin, Jungwirth and MacDonald), confirms that (a) the paired state at $\nu=5 / 2$ and zero tilt is indeed described by the symmetrized MR state (with $98 \%$ weight) and (b) tilting drives the system into a stripe phase. Details of these studies will be given elsewhere.

[1] For reviews, see, e. g. (a) The Quantum Hall Effect, 2nd Ed., edited by R. E. Prange and S. M. Girvin (Springer, New York, 1990); (b) Perspectives in Quantum Hall Effect, edited by S. Das Sarma and A. Pinczuk (Wiley, New York, 1997).

[2] R.L. Willett, M.A. Paalanen, K.W. West, L.N. Pfeiffer, and D.J. Bishop, Phys. Rev. Lett. 65, 112 (1990).

[3] B. I. Halperin, P. A. Lee, and N. Read, Phys. Rev. B 47, 7312 (1993).

[4] J.K. Jain, Phys. Rev. Lett. 63, 199 (1989).

[5] R. Willett, J.P. Eisenstein, H.L. Störmer, D.C. Tsui, A.C. Gossard, and J.H. English, Phys. Rev. Lett. 59, 1776 (1987); see also W. Pan et al, cond-mat/9907356.

[6] M. P. Lilly, K. B. Cooper, J. P. Eisenstein, L. N. Pfeiffer, and K. W. West, Phys. Rev. Lett. 82, 394 (1999).

[7] R. R. Du, D. C. Tsui, H. L. Störmer, L. N. Pfeiffer, K. W. Baldwin, and K. W. West, Solid State Commun. 109, 389 (1999).

[8] M. Shayegan and H.C. Manoharan, cond-mat/9903405.

[9] A. A. Koulakov, M. M. Fogler, and B. I. Shklovskii, Phys. Rev. Lett. 76, 499 (1996); Phys. Rev. B 54, 1853 (1996); M. M. Fogler and A. A. Koulakov, ibid 55, 9326 (1997).

[10] R. Moessner and J. T. Chalker, Phys. Rev. B 54, 5006 (1996).

[11] E.H. Rezayi, F.D.M. Haldane and Kun Yang, Phys. Rev. Lett. 83, 1219 (1999).

[12] F.D.M. Haldane and E. H. Rezayi, Phys. Rev. Lett. 60, 956 (1988); Phys. Rev. Lett. 60, E1886 (1988).

[13] B.I. Halperin, Helv. Phys. Acta, 56, 75 (1983).

[14] G. Moore and N. Read, Nucl. Phys. B360, 362 (1991).

[15] Ref. 12] gives an equivalent form; $(\operatorname{Pf}[A])^{2} \equiv \operatorname{det}[A]$.

[16] M. Greiter, X.-G. Wen and F. Wilczek, Phys. Rev. Lett. 66, 3205 (1991); Nucl. Phys. B 374, 567 (1992).

[17] R.H. Morf, Phys. Rev. Lett. 80, 1505 (1998)

[18] J.P. Eisenstein, R.L. Willett, H.L. Störmer, D.C. Tsui, A.C. Gossard and J.H. English, Phys. Rev. Lett. 61, 997 (1988).

[19] W. Pan, R.R. Du, H.L. Störmer, D.C. Tsui, L.N. Pfeiffer, K.W. Baldwin and K.W. West, Phys. Rev. Lett. 83, 820 (1999).

[20] M.P. Lilly, K.B. Cooper, J.P. Eisenstein, L.N. Pfeiffer and K.W. West, Phys. Rev. Lett. 83, 824 (1999).

[21] F. D. M. Haldane, in Ref. [1] (a), p. 303.

[22] For the Coulomb potential in $n=0 \mathrm{LL}$ these are $V_{m}^{0}=$ $\Gamma(m+1 / 2) /(2 \Gamma(m+1))$, and for $n=1$ they are $V_{m}^{1}=$ $(m-3 / 8)(m-11 / 8) \Gamma(m-3 / 2) /(2 \Gamma(m+1))$.

[23] E. Rezayi and N. Read, Phys. Rev. Lett. 72, 900 (1994).

[24] F. D. M. Haldane et al., unpublished.

[25] F. D. M. Haldane, Phys. Rev. Lett. 55, 2095 (1985).

[26] This is implemented as a PH transformation of the occupation numbers in a "Landau gauge" ( $K_{x}$ eigenstate) ba- 
sis, followed by rotation by $180^{\circ}$ and complex conjugation of the amplitude. This is an anti-unitary operation that leaves $\mathbf{K}$ unchanged; if the $\mathrm{PBC}$ is rectangular (simple or centered), there is also an anti-unitary reflection symmetry which can be combined with the $\mathrm{PH}$ transformation to give a unitary symmetry, under which eigenstates of (3) with reflection-invariant $\mathbf{K}$ are even or odd.

[27] Morf has come to the same conclusion from studies on the sphere where the transition is not as sharp and $V_{1}$ was reduced by $20 \%$ from the "maximum gap" point.

[28] J. P. Eisenstein, in Ref. [1] (b) p. 37.

[29] R. E. Prange, in Ref. [1] (a) p. 1.

[30] K. Park, V. Melik-Alaverdian, N.E. Bonesteel and J.K. Jain, Phys. Rev. B 58, 10167 (1998). 